\title{
Relativistic and Nuclear Structure Effects in Parity-Violating Quasielastic Electron Scattering.
}

\author{
C.J. Horowitz \\ Department of Physics and Nuclear Theory Center, \\ Indiana University, Bloomington, Indiana 47405 \\ J. Piekarewicz \\ Supercomputer Computations Research Institute, \\ Florida State University, Tallahassee, FL 32306
}

\begin{abstract}
The parity violating longitudinal asymmetry $\mathcal{A}$ is calculated for quasielastic electron scattering. We use a variety of relativistic mean field models for the response of nuclear matter and ${ }^{12} \mathrm{C}$ at a momentum transfer of $\mathrm{q}=550 \mathrm{MeV} / \mathrm{c}$. Relativistic effects from a reduced nucleon mass, RPA correlations and vacuum polarization can all change $\mathcal{A}$ by a relatively large amount. These large nuclear structure corrections could make it impossible to extract strange quark matrix elements or radiative corrections to weak axial currents.
\end{abstract}




\section{INTRODUCTION}

Parity violating electron scattering probes weak neutral currents and can provide very interesting information on strange quark matrix elements in nucleons and nuclei. One is interested in the form factors of the the vector (both electric and magnetic) and the axial strange quark currents in a nucleon. These three form factors contain new information on nucleon structure which can help guide and constrain QCD based models.

It will take a number of measurements to determine separately all of these form factors. Furthermore, there are important complications from radiative corrections [1] which hinder the extraction of strange quark matrix elements. Therefore, one anticipates a program of several electron scattering experiments involving proton and different nuclear targets. These results will then be combined with neutrino scattering in order to extract the most accurate strange-quark information.

Clearly one experiment is elastic electron proton scattering with polarized electrons $(\vec{e}, p)$. The parity violating analyzing power (the cross section difference between positive and negative helicity electrons over the sum) is sensitive to weak neutral currents. Indeed, the SAMPLE experiment [2] will place (somewhat crude) limits on the strange quark magnetic form factor.

Neutrino scattering may provide the best information on the axial-vector form factor. For example, the BNL experiment measuring neutrino and antineutrino proton scattering claimed to determine a nonzero strange quark contribution [3]. Note, for experimental reasons, most neutrino experiments measure a combination of elastic scattering from free protons and quasielastic scattering from nucleons bound in nuclei. In a later paper we will examine nuclear structure corrections to quasielastic neutrino scattering and how these could affect the extraction of strange quark matrix elements.

Even if the strange axial current was accurately determined (in neutrino scattering), uncertainties in axial currents could still haunt electron scattering experiments. This is because of radiative corrections to the axial current which are very different (larger) for electrons than for neutrinos [1]. For example, a small "wrong" parity admixture could exist in the nucleon (perhaps due to weak couplings of the nucleon to its meson cloud). The electron could then scatter completely electromagnetically from the small wrong parity admixture. These radiative corrections could be large (since they would not involve the small weak vector coupling of the electron $g_{V}^{e}$ (see Eq. (17c) and Table @). Furthermore, some radiative corrections are difficult to calculate since they involve unknown strong interaction matrix elements.

One alternative is to measure the radiative corrections by performing another experiment (besides elastic electron-proton scattering) which has a different combination of radiative corrections and strange quark contributions. One appealing possibility is to measure parity violation in quasielastic electron scattering. This is favorable experimentally because it has a large cross section and a relatively large asymmetry. Indeed, there has already been one pioneering measurement on ${ }^{9} \mathrm{Be}[$ [ $]$. [However, this experiment was not accurate enough to determine strange quark matrix elements].

Because quasielastic scattering also involves neutrons one measures a different isospin combination than in elastic proton scattering. This could allow one to separate isoscalar strange quark contributions from radiative corrections to the isovector axial current. With 
this in mind, a parity violating quasielastic experiment has been proposed by Donnelly and collaborators [5.,6].

However if a nuclear target is involved, one must immediately address two questions: (a) do unknown nuclear structure effects complicate the extraction of strange quark matrix elements and (b) are the strange quark matrix elements themselves modified in a nucleus? We discuss the second (very interesting) question first.

It is plausible that strange quark currents are different in a nucleus than in a nucleon. For example, the old EMC experiment revealed that up and down quark structure functions are changed by about $10 \%$. One might expect the strange quark content to increase with density. Indeed, in the limit of very high density one expects a transition from nuclear to quark matter. Furthermore, it is believed that the ground state of quark matter (in weak equilibrium) is strange matter containing a large number of strange quarks. Here the large up and down quark Fermi energies overcome the inhibiting effect of the strange quark mass. Even at much lower densities, one can speculate that increasing the up and down quark Fermi energies would help produce more virtual strange-quark pairs. Thus the strange quark content could increase with density.

In a hadronic picture the same conclusion can be reached. Calculations for neutron matter suggest that the fraction of real hyperons increases with density at high density [7]. Furthermore, if a Kaon condensate [8] were to form at high density, the strange quark content would be very large. At lower densities, one could expect the interactions between nucleons and the large nucleon Fermi energy will help drive virtual transitions of nucleons to Kaons and hyperons. Thus the "Kaon cloud" in a nucleus could be larger than in a nucleon. This would increase the strange quark content of nuclear matter.

At present very little is known about the strange quark content of nucleons much less that of a nucleus. Therefore, we will make the minimal assumption that the strange quark matrix elements are the same in a nucleus as in a nucleon. We leave the density dependence of the strange quark contributions as a very interesting question for further work.

In this paper we address the first (more pedestrian but nevertheless crucial) question. How much do nuclear structure uncertainties complicate the extraction of strange quark matrix elements from a quasielastic electron scattering measurement? Because the asymmetry at backward angles involves a ratio of the parity violating weak response to a very similar parity conserving electromagnetic response most nuclear structure effects simply cancel in the ratio. We discuss this in detail below. However, the weak vector current has a very different isospin character from the electromagnetic current. Therefore, one is sensitive to the ratio of isoscalar to isovector responses. A nuclear structure effect that changes the ratio of isoscalar to isovector responses will change the asymmetry and destroy ones ability to extract strange quark matrix elements.

Donnely et al., have argued that this isospin ratio is small and under control (in the transverse response) [5]. [Note, the transverse response dominates over the longitudinal for large electron scattering angles, see below]. Most isoscalar effects are small because of the very small isoscalar anomalous moment of the nucleon. This argument is valid even if there are big random phase approximations (RPA) or other nuclear structure corrections to the bare isoscalar response. The overall isoscalar response is still expected to be small because the electromagnetic probe only couples very weakly through the small isoscalar anomalous moment. 
However, there can be sizable contributions of the Dirac $F_{1}$ form factor to the isoscalar response. In an earlier paper [9], we argued that these contributions could be significantly enhanced by relativistic effects. In the present paper we elaborate on these earlier results and examine RPA and vacuum polarization corrections. Furthermore, we extend earlier nuclear-matter results and present full finite-nucleus responses.

Our formalism for parity violating electron scattering is presented in Sec. II where the asymmetry is expressed as a ratio of several nuclear response functions. Definitions of these response functions are presented in Sec. IIA and results in Sec. III. For simplicity we perform calculations only for ${ }^{12} \mathrm{C}$ and nuclear matter at a momentum transfer of $q=$ $550 \mathrm{MeV} /$ c. Sec. $\mathbb{D}$ presents a summary of our findings and our conclusions. We find that relativistic and other nuclear structure effects are relatively large and that these may destroy the interpretability of a parity violating quasielastic measurement.

\section{FORMALISM}

\section{A. Nuclear Response Functions}

In this section we express the parity violating asymmetry in terms of a number of response functions for weak and electromagnetic currents. This formalism has also been presented in Refs. [5, 9, 10]. The longitudinal analyzing power, or asymmetry, $\mathcal{A}$ is the cross section difference for scattering electrons of positive and negative helicity divided by the sum. It clearly vanishes if parity is conserved. However, in the standard model, interference terms between the weak neutral and the electromagnetic current produce a small but nonzero $\mathcal{A}$.

In lowest order, the inclusive (polarized) cross section

$$
\frac{d^{2} \sigma_{h}}{d \Omega^{\prime} d E^{\prime}} \simeq\left[l_{\mu \nu}^{\gamma \gamma} W_{\gamma \gamma}^{\mu \nu}+a_{0} \tau l_{\mu \nu}^{\gamma Z} W_{\gamma Z}^{\mu \nu}\right]
$$

contains the one-photon exchange contribution plus the $\gamma-Z^{0}$ interference term (the purely weak component is negligible and will not be considered further). The leptonic contribution to the cross section is contained in the two tensors

$$
\begin{aligned}
& l_{\mu \nu}^{\gamma \gamma} \equiv l_{\mu \nu}=\left[k_{\mu} k_{\nu}^{\prime}+k_{\mu}^{\prime} k_{\nu}-g_{\mu \nu}\left(k \cdot k^{\prime}\right)+i h \varepsilon_{\mu \nu \alpha \beta} k^{\alpha} k^{\prime \beta}\right], \\
& l_{\mu \nu}^{\gamma Z} \equiv g_{V}^{e} l_{\mu \nu}+g_{A}^{e} l_{\mu ; \nu 5}=\left(g_{V}^{e}+h g_{A}^{e}\right) l_{\mu \nu} .
\end{aligned}
$$

Here $h$ is the electron helicity and $g_{V}^{e}\left(g_{A}^{e}\right)$ is the weak-vector (weak-axial) charge of the electron (see Table $\mathbb{I}$ ). The response of the nuclear target is contained in the two hadronic tensors $W_{\gamma \gamma}^{\mu \nu}$ and $W_{\gamma z}^{\mu \nu}$. The electromagnetic tensor, defined by

$$
W_{\gamma \gamma}^{\mu \nu}(\mathbf{q}, \omega)=\sum_{n}\left\langle\Psi_{n}\left|\widehat{J}_{E M}^{\mu}(\mathbf{q})\right| \Psi_{0}\right\rangle\left\langle\Psi_{0}\left|\widehat{J}_{E M}^{\nu}(-\mathbf{q})\right| \Psi_{n}\right\rangle \delta\left(\omega-\omega_{n}\right),
$$

can be expressed, using current conservation, Lorentz covariance, and parity invariance, in terms of two independent response functions

$$
W_{\gamma \gamma}^{\mu \nu}=W_{1}\left[g^{\mu \nu}-\frac{q^{\mu} q^{\nu}}{q^{2}}\right]+\frac{W_{2}}{M_{T}^{2}}\left[p^{\mu}-\frac{(p \cdot q)}{q^{2}} q^{\mu}\right]\left[p^{\nu}-\frac{(p \cdot q)}{q^{2}} q^{\nu}\right],
$$


where $q^{\mu} \equiv(\omega, \mathbf{q})$ is the four-momentum transfer to the target, and $p^{\mu}$ and $M_{T}$ are the four-momentum and rest mass of the nuclear target respectively. Since the electromagnetic tensor is symmetric under the exchange of Lorentz $(\mu \leftrightarrow \nu)$ indices, the electromagnetic contribution to the cross section becomes independent of the electron helicity. Furthermore, since weak-interaction effects on the unpolarized cross section are negligible, the parity conserving contribution to the cross section is entirely electromagnetic and is given by,

$$
\frac{1}{\sigma_{M}}\left[\frac{d^{2} \sigma_{h}}{d \Omega^{\prime} d E^{\prime}}\right]_{\mathrm{pc}}=\left[v_{L} S_{L}(\omega, q)+v_{T} S_{T}(\omega, q)\right]
$$

where $\sigma_{M}$ is the Mott cross section, $v_{L}$ and $v_{T}$ are kinematical factors,

$$
v_{L}=\frac{Q^{4}}{\mathbf{q}^{4}} ; \quad v_{T}=\frac{Q^{2}}{2 \mathbf{q}^{2}}+\tan ^{2}(\theta / 2) ; \quad Q^{2} \equiv \mathbf{q}^{2}-\omega^{2},
$$

and $S_{L}$ and $S_{T}$ are the longitudinal and transverse nuclear response functions. These are related to $W_{1}$ and $W_{2}$ by the following simple relations

$$
\begin{aligned}
& S_{L} \equiv W_{\gamma \gamma}^{00}=\frac{\mathbf{q}^{2}}{Q^{2}} W_{1}+\frac{\mathbf{q}^{4}}{Q^{4}} W_{2}, \\
& S_{T} \equiv W_{\gamma \gamma}^{11}+W_{\gamma \gamma}^{22}=-2 W_{1} .
\end{aligned}
$$

The parity-violating electron asymmetry, driven entirely by the electromagnetic-weak interference term, is contained in the hadronic tensor $W_{\gamma Z}^{\mu \nu}$. In order to express the electromagnetic-weak tensor in terms of Lorentz-invariant response functions we write it in terms of vector-vector and vector-axial contributions, i.e.,

$$
W_{\gamma Z}^{\mu \nu} \equiv \widetilde{W}_{\gamma Z}^{\mu \nu}+\widetilde{W}_{\gamma Z}^{\mu ; \nu 5}
$$

where $\widetilde{W}_{\gamma Z}^{\mu \nu}\left(\widetilde{W}_{\gamma Z}^{\mu ; \nu 5}\right)$ arises from the interference of the electromagnetic vector current with the vector(axial) component of the weak current, i.e.,

$$
\begin{aligned}
\widetilde{W}_{\gamma Z}^{\mu \nu}(\mathbf{q}, \omega) & =\sum_{n}\left\langle\Psi_{n}\left|\widehat{J}_{E M}^{\mu}(\mathbf{q})\right| \Psi_{0}\right\rangle\left\langle\Psi_{0}\left|\widehat{J}_{N C}^{\nu}(-\mathbf{q})\right| \Psi_{n}\right\rangle \delta\left(\omega-\omega_{n}\right) \\
\widetilde{W}_{\gamma Z}^{\mu ; \nu 5}(\mathbf{q}, \omega) & =\sum_{n}\left\langle\Psi_{n}\left|\widehat{J}_{E M}^{\mu}(\mathbf{q})\right| \Psi_{0}\right\rangle\left\langle\Psi_{0}\left|\widehat{J}_{N C}^{\nu 5}(-\mathbf{q})\right| \Psi_{n}\right\rangle \delta\left(\omega-\omega_{n}\right) .
\end{aligned}
$$

In particular, the purely vector component has exactly the same Lorentz structure as the electromagnetic tensor displayed in Eq.(州), i.e.,

$$
\widetilde{W}_{\gamma Z}^{\mu \nu}=\widetilde{W}_{1}\left[g^{\mu \nu}-\frac{q^{\mu} q^{\nu}}{q^{2}}\right]+\frac{\widetilde{W}_{2}}{M_{T}^{2}}\left[p^{\mu}-\frac{(p \cdot q)}{q^{2}} q^{\mu}\right]\left[p^{\nu}-\frac{(p \cdot q)}{q^{2}} q^{\nu}\right] .
$$

The vector-axial tensor, on the other hand, must be written in terms of the only available pseudotensor that one can construct from $p^{\mu}$ and $q^{\mu}$, namely,

$$
\widetilde{W}_{\gamma Z}^{\mu ; \nu 5}=-i \frac{\widetilde{W}_{A}}{M_{T}^{2}} \varepsilon^{\mu \nu \alpha \beta} p_{\alpha} q_{\beta}
$$


The parity-violating part of the cross section can now be obtained by performing the contraction of the leptonic tensor $l_{\mu \nu}^{\gamma Z}(\mathrm{Eq} \cdot(2 \mathrm{~b}))$ with the hadronic tensor $W_{\gamma Z}^{\mu \nu}$. This yields,

$$
\frac{1}{\sigma_{M}}\left[\frac{d^{2} \sigma_{h}}{d \Omega^{\prime} d E^{\prime}}\right]_{\mathrm{pv}}=a_{0} \tau h\left[g_{A}^{e}\left(v_{L} \widetilde{S}_{L}(\omega, q)+v_{T} \widetilde{S}_{T}(\omega, q)\right)+g_{V}^{e} v_{T^{\prime}} \widetilde{S}_{A}(\omega, q)\right],
$$

where

$$
v_{T^{\prime}}=\tan (\theta / 2)\left[\frac{Q^{2}}{2 \mathbf{q}^{2}}+\tan ^{2}(\theta / 2)\right]^{1 / 2}
$$

and the three additional nuclear response functions are given by

$$
\begin{aligned}
& \widetilde{S}_{L} \equiv \widetilde{W}_{\gamma Z}^{00}=\frac{\mathbf{q}^{2}}{Q^{2}} \widetilde{W}_{1}+\frac{\mathbf{q}^{4}}{Q^{4}} \widetilde{W}_{2}, \\
& \widetilde{S}_{T} \equiv \widetilde{W}_{\gamma Z}^{11}+\widetilde{W}_{\gamma Z}^{11}=-2 \widetilde{W}_{1}, \\
& \widetilde{S}_{A} \equiv i\left(\widetilde{W}_{\gamma Z}^{1 ; 2(5)}-\widetilde{W}_{\gamma Z}^{2 ; 1(5)}\right)=2 \frac{|\mathbf{q}|}{M_{T}} \widetilde{W}_{A} .
\end{aligned}
$$

The factor $a_{0} \tau\left(\tau \equiv Q^{2} / 4 M^{2}\right)$ sets the scale for the magnitude of the parity-violating effects and is given by,

$$
a_{0}=-\frac{G_{F} M^{2}}{\sqrt{2} \pi \alpha} \simeq-3.172 \times 10^{-4}
$$

Notice that in the above expression only terms linear in the electron helicity were kept. Terms independent of the electron helicity make a negligible contribution to the parityconserving cross section and were neglected. The parity-violating asymmetry, defined as the difference of helicity cross sections divided by their sum, is now given by

$$
\mathcal{A}=\frac{d \sigma_{\uparrow}-d \sigma_{\downarrow}}{d \sigma_{\uparrow}+d \sigma_{\downarrow}}=\mathcal{A}_{L}+\mathcal{A}_{T}+\mathcal{A}_{T^{\prime}}
$$

where

$$
\begin{aligned}
& \mathcal{A}_{L} / a_{0} \tau=g_{A}^{e} \frac{v_{L} \widetilde{S}_{L}}{v_{L} S_{L}+v_{T} S_{T}}, \\
& \mathcal{A}_{T} / a_{0} \tau=g_{A}^{e} \frac{v_{T} \widetilde{S}_{T}}{v_{L} S_{L}+v_{T} S_{T}}, \\
& \mathcal{A}_{T^{\prime}} / a_{0} \tau=g_{V}^{e} \frac{v_{T^{\prime}} \widetilde{S}_{A}}{v_{L} S_{L}+v_{T} S_{T}} .
\end{aligned}
$$

The hadronic tensors defined above are intimately related to a fundamental many-body operator, namely, the current-current correlation function or polarization tensor. This relation can be illustrated, for example, in the case of the timelike-timelike component of the electromagnetic tensor,

$$
W_{\gamma \gamma}^{00}=-\frac{1}{\pi} \mathcal{I} m \sum_{n} \frac{\left\langle\Psi_{0}\left|\widehat{J}_{E M}^{0}(-q)\right| \Psi_{n}\right\rangle\left\langle\Psi_{n}\left|\widehat{J}_{E M}^{0}(q)\right| \Psi_{0}\right\rangle}{\omega-\omega_{n}+i \eta}=-\frac{1}{\pi} \mathcal{I} m \Pi_{\gamma \gamma}^{00}(\mathbf{q}, \omega),
$$


where the polarization tensor, $\Pi_{\gamma \gamma}^{00}$, has been introduced in the last line. Consequently, all five nuclear response functions can be written in terms of appropriate components of several polarization tensors, i.e.,

$$
\begin{aligned}
& S_{L}(\mathbf{q}, \omega)=-\frac{1}{\pi} \mathcal{I} m \Pi_{\gamma \gamma}^{00}(\mathbf{q}, \omega) \\
& S_{T}(\mathbf{q}, \omega)=-\frac{1}{\pi} \mathcal{I} m\left(\Pi_{\gamma \gamma}^{11}(\mathbf{q}, \omega)+\Pi_{\gamma \gamma}^{22}(\mathbf{q}, \omega)\right), \\
& \widetilde{S}_{L}(\mathbf{q}, \omega)=-\frac{1}{\pi} \mathcal{I} m \Pi_{\gamma Z}^{00}(\mathbf{q}, \omega), \\
& \widetilde{S}_{T}(\mathbf{q}, \omega)=-\frac{1}{\pi} \mathcal{I} m\left(\Pi_{\gamma Z}^{11}(\mathbf{q}, \omega)+\Pi_{\gamma Z}^{22}(\mathbf{q}, \omega)\right), \\
& \widetilde{S}_{A}(\mathbf{q}, \omega)=-\frac{1}{\pi} \mathcal{I} m\left(i \Pi_{\gamma Z}^{1 ; 2(5)}(\mathbf{q}, \omega)-i \Pi_{\gamma Z}^{2 ; 1(5)}(\mathbf{q}, \omega)\right) .
\end{aligned}
$$

The advantage of relating all nuclear response functions to a many-body operator, i.e., the polarization tensor, is that the responses can be systematically computed using wellknown many-body techniques (e.g., Feynman diagrams). In particular, this will enable us to calculate a nuclear response that goes beyond the simple impulse, or uncorrelated, response by including many-body correlation effects.

The various nuclear response functions can, then, be calculated from imaginary parts of polarization insertions given a model for: (a) the hadronic weak and electromagnetic currents and (b) the nuclear structure. Hadronic currents are discussed in the next section. Subsequently, we calculate the nuclear responses in a number of relativistic mean-field models with and without RPA correlations and with various treatments of vacuum polarization.

\section{B. Electromagnetic and Weak Hadronic Currents}

In this section we describe the electromagnetic and weak hadronic currents that will be used in calculating the five nuclear response functions. We start by writing the electromagnetic current for the three lightest $(u, d, s)$ quarks

$$
J_{E M}^{\mu}=e_{u} \bar{u} \gamma^{\mu} u+e_{d} \bar{d} \gamma^{\mu} d+e_{s} \bar{s} \gamma^{\mu} s
$$

For two quark flavors $(u$ and $d$ ) one customarily rewrites the electromagnetic current in terms of isoscalar and isovector contributions. The analogous expression in the three-flavor case is given in terms of two octet (3 and 8) and the singlet $\mathrm{SU}(3)$ currents, i.e.,

$$
\begin{aligned}
J_{E M}^{\mu} & =e_{0} \bar{q} \gamma^{\mu} q+e_{3} \bar{q} \gamma^{\mu} \frac{\lambda_{3}}{2} q+e_{8} \bar{q} \gamma^{\mu} \frac{\lambda_{8}}{2} q \\
& =\bar{q} \gamma^{\mu} \frac{\lambda_{3}}{2} q+\frac{1}{\sqrt{3}} \bar{q} \gamma^{\mu} \frac{\lambda_{8}}{2} q .
\end{aligned}
$$

where we have defined (and used) singlet and octet electromagnetic charges

$$
\begin{aligned}
& e_{0}=\left(e_{u}+e_{d}+e_{s}\right) / 3, \\
& e_{3}=\left(e_{u}-e_{d}\right), \\
& e_{8}=\left(e_{u}+e_{d}-2 e_{s}\right) / \sqrt{3} .
\end{aligned}
$$


Single-nucleon electromagnetic form factors are now obtained by evaluating the electromagnetic current between nucleon states i.e.,

$$
\begin{aligned}
\left\langle N\left(p^{\prime} s^{\prime} t^{\prime}\right)\right| J_{E M}^{\mu}(T & =0)|N(p s t)\rangle=\frac{1}{\sqrt{3}}\left\langle N\left(p^{\prime} s^{\prime} t^{\prime}\right)\left|\bar{q} \gamma^{\mu} \frac{\lambda_{8}}{2} q\right| N(p s t)\right\rangle \\
& =\bar{U}\left(p^{\prime} s^{\prime}\right)\left[F_{1}^{(0)} \gamma^{\mu}+i F_{2}^{(0)} \sigma^{\mu \nu} \frac{q_{\nu}}{2 M}\right] U(p, s) \delta_{t^{\prime} t}, \\
\left\langle N\left(p^{\prime} s^{\prime} t^{\prime}\right)\right| J_{E M}^{\mu}(T & =1)|N(p s t)\rangle=\left\langle N\left(p^{\prime} s^{\prime} t^{\prime}\right)\left|\bar{q} \gamma^{\mu} \frac{\lambda_{3}}{2} q\right| N(p s t)\right\rangle \\
& =\bar{U}\left(p^{\prime} s^{\prime}\right)\left[F_{1}^{(1)} \gamma^{\mu}+i F_{2}^{(1)} \sigma^{\mu \nu} \frac{q_{\nu}}{2 M}\right] U(p, s)\left(\tau_{3}\right)_{t^{\prime} t} .
\end{aligned}
$$

The weak-vector and weak-axial neutral currents can be written in analogy to Eq. (21). First, however, one rewrites the SU(3)-singlet current in terms of one octet (eight) and a pure strange-quark current. This yields,

$$
J_{N C}^{\mu}=\xi_{V}^{(1)} \bar{q} \gamma^{\mu} \frac{\lambda_{3}}{2} q+\xi_{V}^{(0)} \bar{q} \gamma^{\mu} \frac{\lambda_{8}}{2} q+\xi_{V}^{(s)} \bar{s} \gamma^{\mu} s
$$

where the couplings are simple linear combinations of quark weak-vector charges (see Table III), i.e.,

$$
\begin{aligned}
& \xi_{V}^{(0)}=\sqrt{3}\left(g_{V}^{u}+g_{V}^{d}\right) \\
& \xi_{V}^{(1)}=\left(g_{V}^{u}-g_{V}^{d}\right) \\
& \xi_{V}^{(s)}=\left(g_{V}^{u}+g_{V}^{d}+g_{V}^{s}\right) .
\end{aligned}
$$

Writing the hadronic current as above, enables one to express nucleon matrix elements of the weak-vector current in terms of electromagnetic isoscalar and isovector form factors plus a strange-quark contribution (see Eqs. (23a, 23a)),

$$
\begin{aligned}
\left\langle N^{\prime}\left|J_{N C}^{\mu}(T=0)\right| N\right\rangle & =\sqrt{3} \xi_{V}^{(0)}\left\langle N^{\prime}\left|J_{E M}^{\mu}(T=0)\right| N\right\rangle+\xi_{V}^{(s)}\left\langle N^{\prime}\left|\bar{s} \gamma^{\mu} s\right| N\right\rangle, \\
\left\langle N^{\prime}\left|J_{N C}^{\mu}(T=1)\right| N\right\rangle & =\xi_{V}^{(1)}\left\langle N^{\prime}\left|J_{E M}^{\mu}(T=1)\right| N\right\rangle
\end{aligned}
$$

where we have assumed that matrix elements of the strange-quark current are equal for protons and neutrons. Defining single-nucleon strange $\left(F_{1}^{(s)}, F_{2}^{(s)}\right)$ and weak-vector form factors $\left(\widetilde{F}_{1}^{(0)}, \widetilde{F}_{2}^{(0)}, \widetilde{F}_{1}^{(1)}, \widetilde{F}_{2}^{(1)}\right)$ in analogy to Eqs. (23a, 23b) we arrive at the following useful relations between single-nucleon form factors

$$
\begin{array}{lll}
\widetilde{F}_{1}^{(0)}=\sqrt{3} \xi_{V}^{(0)} F_{1}^{(0)}+\xi_{V}^{(s)} F_{1}^{(s)}, & \widetilde{F}_{1}^{(1)}=\xi_{V}^{(1)} F_{1}^{(1)}, \\
\widetilde{F}_{2}^{(0)}=\sqrt{3} \xi_{V}^{(0)} F_{2}^{(0)}+\xi_{V}^{(s)} F_{2}^{(s)}, & \widetilde{F}_{2}^{(1)}=\xi_{V}^{(1)} F_{2}^{(1)} .
\end{array}
$$

Finally, one defines, in analogy to Eq. (24), the weak-axial neutral current in terms of octet and strange axial-vector currents and the appropriate weak-axial charges (see Table [II)

$$
J_{N C}^{\mu 5}=\xi_{A}^{(1)} \bar{q} \gamma^{\mu} \gamma^{5} \frac{\lambda_{3}}{2} q+\xi_{A}^{(0)} \bar{q} \gamma^{\mu} \gamma^{5} \frac{\lambda_{8}}{2} q+\xi_{A}^{(s)} \bar{s} \gamma^{\mu} \gamma^{5} s
$$

Single-nucleon form factors are obtained (neglecting induced-pseudoscalar contributions) by evaluating octet and strange axial-vector currents between nucleon states 


$$
\begin{aligned}
\left\langle N\left(p^{\prime} s^{\prime} t^{\prime}\right)\left|\bar{q} \gamma^{\mu} \gamma^{5} \frac{\lambda_{3}}{2} q\right| N(p s t)\right\rangle & =G_{A}^{(3)}\left[\bar{U}\left(p^{\prime} s^{\prime}\right) \gamma^{\mu} \gamma^{5} U(p, s)\right]\left(\tau_{3}\right)_{t^{\prime} t} \\
\left\langle N\left(p^{\prime} s^{\prime} t^{\prime}\right)\left|\bar{q} \gamma^{\mu} \gamma^{5} \frac{\lambda_{8}}{2} q\right| N(p s t)\right\rangle & =G_{A}^{(8)}\left[\bar{U}\left(p^{\prime} s^{\prime}\right) \gamma^{\mu} \gamma^{5} U(p, s)\right] \delta_{t^{\prime} t} \\
\left\langle N\left(p^{\prime} s^{\prime} t^{\prime}\right)\left|\bar{s} \gamma^{\mu} \gamma^{5} s\right| N(p s t)\right\rangle & =G_{A}^{(s)}\left[\bar{U}\left(p^{\prime} s^{\prime}\right) \gamma^{\mu} \gamma^{5} U(p, s)\right] \delta_{t^{\prime} t}
\end{aligned}
$$

Isoscalar and isovector single-nucleon axial form factors are now obtained from the following relations

$$
\widetilde{G}_{A}^{(0)}=\xi_{A}^{(0)} G_{A}^{(8)}+\xi_{A}^{(s)} G_{A}^{(s)}, \quad \widetilde{G}_{A}^{(1)}=\xi_{A}^{(1)} G_{A}^{(3)} .
$$

In conclusion, the electromagnetic and weak-neutral currents that one is to employ in calculating all nuclear response functions have been written in terms of appropriate singlenucleon form factors (described in the appendix) and are given by,

$$
\begin{aligned}
\widehat{J}_{E M}^{\mu} & =\left[F_{1}^{(0)} \gamma^{\mu}+i F_{2}^{(0)} \sigma^{\mu \nu} \frac{q_{\nu}}{2 M}\right]+\left[F_{1}^{(1)} \gamma^{\mu}+i F_{2}^{(1)} \sigma^{\mu \nu} \frac{q_{\nu}}{2 M}\right] \tau_{z}, \\
\widehat{J}_{N C}^{\mu} & =\left[\widetilde{F}_{1}^{(0)} \gamma^{\mu}+i \widetilde{F}_{2}^{(0)} \sigma^{\mu \nu} \frac{q_{\nu}}{2 M}\right]+\left[\widetilde{F}_{1}^{(1)} \gamma^{\mu}+i \widetilde{F}_{2}^{(1)} \sigma^{\mu \nu} \frac{q_{\nu}}{2 M}\right] \tau_{z}, \\
\widehat{J}_{N C}^{\mu 5} & =\widetilde{G}_{A}^{(0)} \gamma^{\mu} \gamma^{5}+\widetilde{G}_{A}^{(1)} \gamma^{\mu} \gamma^{5} \tau_{z} .
\end{aligned}
$$

The above equations are a major assumption of our model and prescribe how the hadronic currents are taken "off-shell". We also assume that the form factors are unchanged in the medium.

\section{Relativistic Mean-Field Responses}

In this section we describe the model used in the calculation of the various weak and electromagnetic response functions. Our starting point is a relativistic mean field approximation to the Walecka model where nucleons move in strong scalar $\Sigma_{\mathrm{s}}$ and vector $\Sigma_{\mathrm{v}}$ mean fields [12,[13]. In particular, the strong scalar field is responsible for reducing the nucleon mass from its free-space value to $M^{*}$

$$
M^{*} \equiv M-\Sigma_{\mathrm{s}} .
$$

For nuclear matter this is a constant that must be self-consistently determined at every given density. In a finite nucleus, however, the effective mass depends on position. It is smaller at the center and rises back to its free value $M$ at the surface.

These mean field models provide perhaps the minimal relativistic description of a nuclear target. The strong mean fields are very closely related to the spin-orbit potential of the shell model. Furthermore, $M^{*}$ provides a minimal description of the binding energy shift seen in the position of the quasielastic peak [11.

We illustrate the formalism by presenting electromagnetic, weak vector and weak axial response functions for nuclear matter. Most of the formalism, however, remains valid in the finite system. The appropriate modifications to be carried out for a finite nucleus and for including RPA correlations will be discussed at the end of the section. 
The basic ingredient in calculating all nuclear response functions is the nucleon Green's function (or Feynman propagator). The Green's function describes the propagation of nucleons in the mean fields. In a self-consistent Hartree approximation the nucleon propagator is given by [13]

$$
G(k)=\left[\not k+M^{*}\right]\left(\frac{1}{k^{2}-M^{* 2}+i \epsilon}+i \frac{\pi}{E_{k}^{*}} \delta\left(k^{0}-E_{k}^{*}\right) \Theta\left(k_{F}-|\mathbf{k}|\right)\right) \equiv G_{\mathrm{F}}+G_{\mathrm{D}},
$$

where $E_{k}^{*}=\sqrt{\mathbf{k}^{2}+M^{* 2}}$ and $k_{F}$ is the Fermi momentum. $G_{\mathrm{F}}$ is that part of the propagator having the same analytic structure as the free Feynman propagator. The density-dependent part of the propagator, $G_{\mathrm{D}}$, corrects $G_{\mathrm{F}}$ for the presence of occupied states below the Fermi surface.

The electromagnetic (Eq.(31a)) and weak-vector currents (Eq.(31b)) have been written in terms of Dirac and Pauli (or anomalous) contributions. This implies, that the electromagnetic $\left(\Pi_{\gamma \gamma}^{\mu \nu}\right)$ as well as the weak-vector $\left(\Pi_{\gamma Z}^{\mu \nu}\right)$ polarization tensors will have to be evaluated in terms of individual polarizations having Lorentz vector $\mathrm{v}=\gamma^{\mu}$ and tensor $\mathrm{t}=\sigma^{\mu \nu}$ vertices, i.e., (ignoring isospin labels)

$$
\begin{aligned}
i \Pi_{\mathrm{vv}}^{\mu \nu} & =\int \frac{d^{4} k}{(2 \pi)^{4}} \operatorname{Tr}\left[G(k) \gamma^{\mu} G(k+q) \gamma^{\nu}\right], \\
i \Pi_{\mathrm{vt}}^{\mu \nu} & =\int \frac{d^{4} k}{(2 \pi)^{4}} \operatorname{Tr}\left[G(k) \gamma^{\mu} G(k+q) \frac{i \sigma^{\nu \rho} q_{\rho}}{2 M}\right], \\
i \Pi_{\mathrm{tt}}^{\mu \nu} & =\int \frac{d^{4} k}{(2 \pi)^{4}} \operatorname{Tr}\left[G(k) \frac{\sigma^{\mu \eta} q_{\eta}}{2 M} G(k+q) \frac{\sigma^{\nu \rho} q_{\rho}}{2 M}\right] .
\end{aligned}
$$

Note that, both, the electromagnetic and the weak-vector responses are driven by the same polarizations. The only difference between the two responses arises from the use of either electromagnetic or weak-vector single-nucleon form factors. One might expect most nuclear structure effects to change Eqs. (34a, 34b, 34d) in similar ways. Indeed, within a model, one could try to combine the three polarizations and express the responses in terms of the electric and magnetic form factors. However, we chose to define our off-shell currents directly from Eqs. (31a, 31b) in terms of $F_{1}$ and $F_{2}$ form factors. Indeed, we find (in the next section) different relativistic effects on $\prod_{\mathrm{vv}}^{\mu \nu}$ than on $\prod_{\mathrm{tt}}^{\mu \nu}$. Thus, in our model, it is important to break the response up into these three pieces (vv, vt, and tt).

The weak-axial polarization, $\Pi_{\gamma z}^{\mu ; \nu 5}$, can be similarly written in terms of polarizations having either a vector or a tensor vertex, and an axial-vector vertex $\mathrm{a}=\gamma^{\mu} \gamma^{5}$,

$$
\begin{aligned}
i \Pi_{\mathrm{va}}^{\mu ; \nu 5} & =\int \frac{d^{4} k}{(2 \pi)^{4}} \operatorname{Tr}\left[G(k) \gamma^{\mu} G(k+q) \gamma^{\nu} \gamma^{5}\right], \\
i \Pi_{\mathrm{ta}}^{\mu ; \nu 5} & =\int \frac{d^{4} k}{(2 \pi)^{4}} \operatorname{Tr}\left[G(k) \frac{i \sigma^{\mu \eta}(-q)_{\eta}}{2 M} G(k+q) \gamma^{\nu} \gamma^{5}\right] .
\end{aligned}
$$

Note, that the Green's function defined in Eq. 33, describes the propagation of both nucleons and antinucleons. Hence, the above polarization tensors contain, in addition to the usual particle-hole excitations, nucleon-antinucleon excitations (vacuum polarization). However, vacuum polarization has no imaginary part in the space-like region $(q>\omega)$ probed 
in electron scattering. Therefore, vacuum polarization makes no contribution to the uncorrelated responses. Vacuum polarization will, however, make an important contribution to the correlated response that will be presented in Sec. [II].

Using the above defined polarizations together with the single-nucleon form factors leads to the following expressions for the electromagnetic and electromagnetic-weak polarization tensors

$$
\begin{aligned}
\Pi_{\gamma \gamma}^{\mu \nu(i)} & =F_{1}^{(i)} F_{1}^{(i)} \Pi_{\mathrm{vv}}^{\mu \nu(i)}+\left(F_{1}^{(i)} F_{2}^{(i)}+F_{2}^{(i)} F_{1}^{(i)}\right) \Pi_{\mathrm{vt}}^{\mu \nu(i)}+F_{2}^{(i)} F_{2}^{(i)} \Pi_{\mathrm{tt}}^{\mu \nu(i)}, \\
\Pi_{\gamma Z}^{\mu \nu(i)} & =F_{1}^{(i)} \widetilde{F}_{1}^{(i)} \Pi_{\mathrm{vv}}^{\mu \nu(i)}+\left(F_{1}^{(i)} \widetilde{F}_{2}^{(i)}+F_{2}^{(i)} \widetilde{F}_{1}^{(i)}\right) \Pi_{\mathrm{vt}}^{\mu \nu(i)}+F_{2}^{(i)} \widetilde{F}_{2}^{(i)} \Pi_{\mathrm{tt}}^{\mu \nu(i)}, \\
\Pi_{\gamma Z}^{\mu, \nu 5(i)} & =F_{1}^{(i)} \widetilde{G}_{A}^{(i)} \Pi_{\mathrm{va}}^{\mu j \nu(i)}+F_{2}^{(i)} \widetilde{G}_{A}^{(i)} \Pi_{\mathrm{ta}}^{\mu, j \nu 5(i)} .
\end{aligned}
$$

To summarize, the response functions depend on a variety of nucleon electromagnetic and weak form factors. These form factors are collected in the Appendix. The nuclear structure, on the other hand, is contained in the polarization insertions $\prod_{\mathrm{vv}}^{\mu \nu}, \prod_{\mathrm{vt}}^{\mu \nu}, \prod_{\mathrm{tt}}^{\mu \nu}, \Pi_{\mathrm{va}}^{\mu j \nu 5}$, and $\prod_{\mathrm{ta}}^{\mu ; \nu 5}$.

The response functions can be calculated in various approximations. The simplest approximation is to calculate uncorrelated response functions for nuclear matter. This can be done either for $M^{*}=M$, which gives the free Fermi gas results, or for a smaller value of $M^{*}$. These results were presented in Ref. 99 and are included below for comparison. Note that in these approximations the isoscalar and isovector polarizations are identical. The change in the responses arises exclusively from the difference in isoscalar and isovector single-nucleon form factors.

Alternatively, one can calculate the response functions directly in a finite nucleus and avoid a local density approximation. The finite-nucleus calculations can be carried out using a nonspectral representation of the nucleon Green's function as discussed in Refs. [14, 15, 16]. Next, long range correlations can be included by calculating RPA polarization insertions Refs. [14, 15, 16, 17]. We employ a residual interaction consisting of only isoscalar sigma and omega meson exchange with self-consistent parameters (see Table ПII) chosen to fit ground-state properties [18]. In particular note that the isovector response is insensitive to correlations and thus remains identical to its uncorrelated value. Finally, vacuum polarization can be included, in addition to particle-hole or core polarization, in calculating the RPA response. Further details can be found in Ref. [16].

\section{RESULTS}

We now present response functions for the $\mathrm{N}=\mathrm{Z}$ target ${ }^{12} \mathrm{C}$. We expect similar results for heavier nuclei. For simplicity we consider only a momentum transfer of $q=550 \mathrm{MeV} /$ c. Traditional nuclear structure uncertainties will be larger for much smaller momentum transfers. We expect similar relativistic effects for momentum transfers greater than $q=550 \mathrm{MeV} / \mathrm{c}$, but this remains to be investigated.

First we notice that the parity-violating asymmetry $\mathcal{A}$ is dominated by the transverse weak vector contribution $\mathcal{A}_{T}$ with $\mathcal{A}_{T^{\prime}}$ making perhaps a 20 percent contribution for large scattering angles and $\mathcal{A}_{L}$ contributing perhaps 10 percent at forward angles. Furthermore, $\mathcal{A}_{T}$ is itself dominated by the isovector response from the $F_{2}^{(1)}$ form factor contributions (last term in Eq. (36b)). 
For a large-angle experiment, say at an electron scattering angle of 150 degrees, $\mathcal{A}_{L}$ is unimportant (as is the contribution from the longitudinal response $S_{L}$ to the parityconserving cross section) because of the kinematic factors $v_{L}$ and $v_{T}\left(v_{L} / v_{T} \rightarrow 0\right.$ at backward angles). Thus, let us focus on $\mathcal{A}_{T}$. We will return to the small $\mathcal{A}_{T^{\prime}}$ contribution at the end. Furthermore, we will neglect strange-quark contributions so that, from Eqs. (27a and 27b), $\widetilde{F}_{i}^{(0)}=\sqrt{3} \xi_{V}^{(0)} F_{i}^{(0)}$ and $\widetilde{F}_{i}^{(1)}=\xi_{V}^{(1)} F_{i}^{(1)}$. Thus $\mathcal{A}_{T}$ only depends on the ratio of isoscalar $S_{T}^{(0)}$ to isovector $S_{T}^{(1)}$ electromagnetic responses. The only nuclear structure that $\mathcal{A}_{T}$ is sensitive to is isospin. At large angles then, $\mathcal{A}_{T}$ becomes (neglecting the contribution from strange quarks)

$$
\mathcal{A}_{T}=g_{A}^{e} \xi_{V}^{(1)}\left[\frac{1+\frac{\sqrt{3} \xi_{V}^{(0)}}{\xi_{V}^{(1)}} \mathcal{R}_{T}}{1+\mathcal{R}_{T}}\right],
$$

where the isospin ratio is given by,

$$
\mathcal{R}_{T} \equiv S_{T}^{(0)} / S_{T}^{(1)} .
$$

The couplings $\sqrt{3} \xi_{V}^{(0)}$ and $\xi_{V}^{(1)}$ (Table 【1) have opposite signs and almost equal magnitude. Therefore a change in the ratio $\mathcal{R}_{T}$ by 0.01 (i.e., $\mathcal{R}_{T} \rightarrow \mathcal{R}_{T} \pm 0.01$ ) will change $\mathcal{A}_{T}$ by about two percent. However, the total transverse response $\left(S_{T}^{(0)}+S_{T}^{(1)}\right)$ is predominantly isovector because of the large isovector anomalous moment of the nucleon. Thus $\mathcal{R}_{T}$ is expected to be small (of the order of $5 \%$ ). However, one must investigate the nuclear structure uncertainties in $\mathcal{R}_{T}$. Most traditional corrections to the isoscalar response are hidden because of the small isoscalar anomalous moment of the nucleon.

However, there could be large relativistic corrections to the isoscalar transverse response $S_{T}^{(0)}$. A smaller value of $M^{*}$ enhances $\Pi_{\mathrm{vv}}^{11}$ and thus increases $S_{T}^{(0)}$. [Note, $S_{T}^{(0)}$ is dominated by $\Pi_{\mathrm{vv}}^{11}$ rather than by $\Pi_{\mathrm{tt}}^{11}$ because of the small value for $F_{2}^{(0)}$.] The smaller value of $M^{*}$ enhances a nucleon's velocity and produces a larger convection current (which is half isoscalar). This could lead to a large change in $\mathcal{A}_{T}$.

Below, we present results showing that the uncertainties in the transverse response, and hence on $\mathcal{A}_{T}$, are large. For simplicity we do not show results for longitudinal responses. However, most of these have already been published in Ref. [16]. It is already generally agreed that nuclear structure uncertainties in the longitudinal response (and hence in $\mathcal{A}_{L}$ ) are large.

We present a catalog of transverse responses for ten different calculations. First, we consider four different finite nucleus calculations. Uncorrelated (or impulse) and RPA responses are shown first neglecting vacuum polarization in a so called mean field theory (MFT) and then with vacuum polarization in a relativistic Hartree approximation (RHA). For comparison we also show the corresponding four responses calculated in nuclear matter. This allows one to examine commonly made local density approximations. Finally, we also show two "nonrelativistic" calculations; one with and the other without RPA correlations. Here, a Fermi gas is considered with an effective mass equal to the free nucleon mass. In Table [II] we list both sets of parameters used in the calculations.

Ten calculations lead to somewhat complicated results. However, there are important motivations to examine RPA, vacuum polarization and full finite nucleus corrections to 
simple local density approximation results. First, relativistic effects for magnetic moments, seen in impulse approximations, vanish in full RPA calculations [20,21]. Do the relativistic effects for $\mathcal{A}_{T}$ claimed in Ref. 9] also vanish in RPA? Below, we see that they do not.

Next, vacuum polarization may have an important effect on the unpolarized longitudinal response [16,22, 23. As the effective nucleon mass decreases it is easier to excite virtual nucleon-antinucleon pairs out of the vacuum. Therefore, the vacuum becomes better at screening charge. This change in vacuum polarization with $M^{*}$ reduces the charge visible to the electron probe. Relativistic RPA calculations including vacuum polarization have longitudinal responses reduced by about twenty five percent compared to impulse approximations. This reduction is in good agreement with experiment for medium and heavy nuclei (although the experimental errors are large). In contrast, nonrelativistic calculations have integrated responses constrained by the Coulomb sum rule and over predict the data. Below we examine the effect of vacuum polarization on parity violation.

Finally, many quasielastic calculations are done in a local density approximation (LDA). This is expected to be qualitatively correct for the high excitation energies of quasifree scattering. However, we check to see if parity violation is sensitive to any of the details of the response not reproduced by a LDA. In particular the high and low excitation energy parts of the response are poorly described in LDA.

Figure 1 shows transverse response functions calculated in nuclear matter compared to data from ${ }^{12} \mathrm{C}$ at a momentum transfer of $q=550 \mathrm{MeV} / \mathrm{c}$. We consider an average density of ${ }^{12} \mathrm{C}$ which corresponds to a Fermi momentum of $k_{F}=225 \mathrm{MeV} / \mathrm{c}$. First, free Fermi gas results are shown with $M^{*}=M$. These are equivalent to the results reported in Ref. [5]. Next, relativistic MFT results are shown, as in Ref. [9], with an effective mass of $M^{*}=0.68 M$. This is the self-consistent value for the MFT at a density corresponding to $k_{F}=225 \mathrm{MeV} / \mathrm{c}$. The primary effect of $M^{*}$ is to shift the strength to higher energies in agreement with data. Of course there is still substantial experimental strength at medium to high excitation energies. Presumably this corresponds to delta and pion production, multinucleon knockout and or meson exchange currents. These effects are outside the scope of the one-body mechanism assumed for all of our calculations. We should mention that no attempt was made to fit the data by selecting an optimal value for $k_{F}$. As we will show below finite-nucleus calculations give a more realistic distribution of transverse strength.

The contribution of other mechanisms to the quasifree response is an important source of uncertainty for parity violating experiments. At this time it is not well known the relative importance of different processes much less their isoscalar vs. isovector contribution. One might argue that Delta or pion exchange currents are isovector. However, there could be isoscalar contributions from other mesons or from two-nucleon correlations. Any effect from these other contributions will only strengthen our conclusion that there are important nuclear structure uncertainties in parity violating quasielastic electron scattering.

We also show RPA responses in Fig. 1. These use a simple isoscalar interaction consisting of sigma and omega meson exchange. The parameters are taken from the MFT fit to the saturation density and binding energy of nuclear matter [12,13. We assume that the isovector interaction is zero. Consequently, since the total response is dominated by the isovector part, RPA has almost no effect on the unpolarized transverse response.

Next, in Fig. 2 we show the isoscalar contribution to the transverse response. In principle, this response contributes to the observable unpolarized cross section. However, because it 
is so small (compare the scales of Figs. 11 and 2) it is essentially unconstrained by data. As $M^{*}$ decreases the isoscalar response is predicted to be significantly enhanced. This effect was discussed in Ref. [9].

The RPA correlations are seen to shift strength downward (due to an attractive particlehole interaction) and somewhat reduce the area. This is true in both the MFT and $M^{*}=M$ calculations. Note, there is no simple relation between the MFT RPA and the free Fermi-gas results. This is in contrast to the situation for (isoscalar) magnetic moments where there was a Fermi liquid theory theorem constraining the results 20,21. Instead, the quasifree response at finite momentum transfer is a dynamical quantity and depends on the details of the model. There is no theorem which constrains the finite- $q$ response.

The ratio $\mathcal{R}_{T}$ of isoscalar to isovector transverse responses is shown in Fig. 3. This is the only nuclear structure information needed to determine $\mathcal{A}_{T}$ (see Eq. (37)). In general there is a large enhancement in this ratio for the MFT calculations. However, there are also large effects from RPA correlations in both the MFT and $M^{*}=M$ calculations. Figure 3 also shows $\mathcal{A}_{T}$ deduced from $\mathcal{R}_{T}$ by using Eq. (37). This quantity is directly related to the experimental asymmetry. The 5 to 10 percent spread in $\mathcal{A}_{T}$ is much larger than the perhaps one to two percent accuracy [5] needed to measure strange quark effects or radiative corrections to the axial contributions.

We now consider full finite nucleus calculations for ${ }^{12} \mathrm{C}$. Our finite nucleus calculations have been described in Ref. [16] and are based on calculating the nucleon Green's function directly in the finite system. The RPA integral equations are then solved by matrix inversion in momentum space. The interaction parameters are the same ones used to fit ground-state properties so the calculations explicitly conserve current.

Figure 1 shows MFT results for the total transverse response. First, the finite nucleus calculations are qualitatively similar to the nuclear matter results. However, the shift in the position of the peak and the broadening of the width are not as pronounced as in the nuclear matter case. In addition, there is a high energy tail in the finite nucleus response which is not reproduced by the nuclear matter calculations. Figure 5 shows isoscalar responses. Again, the finite nucleus responses have a high energy tail not present in nuclear matter. However, the LDA results are qualitatively similar for the lower energies.

The isospin ratio $\mathcal{R}_{T}$ is shown in Fig. 6. This is qualitatively similar in finite nucleus and LDA calculations. However, the uncorrelated finite nucleus calculations predict an enhancement in $\mathcal{R}_{T}$ at high excitation energies. This is due to the different energy dependences of $\Pi_{\mathrm{vv}}^{11}$ (which is dominant for the isoscalar response) and $\Pi_{\mathrm{tt}}^{11}$ (which dominates the isovector) in the finite nucleus results. Note, we have not yet investigated the origin of this difference. The $\mathcal{A}_{T}$ deduced from these ratios are also shown in Fig. 6. Again there is a 5 to 10 percent dispersion in the results.

Finally, we consider RHA calculations which include vacuum polarization [16,22]. Both nuclear matter and finite nucleus RHA results are shown in Figs. (7, 8, 9). The most important difference between these curves and MFT results may not be intrinsic to vacuum polarization. Instead, the RHA has a somewhat larger effective mass. This is because the RHA includes a term describing the change in the energy density of the vacuum (with $M^{*}$ ). This term tends to resist changes in the nucleon mass. [Also, the RHA reproduces nuclear matter saturation with somewhat smaller meson couplings]. Therefore, RHA results tend to be intermediate between MFT and $M^{*}=M$ calculations. In particular, the isoscalar 
response is not enhanced as much in the RHA as it is in the MFT.

In summary we have presented results for $\mathcal{A}_{T}$ for ten different calculations in Figs. (3), 6 and 9). Depending on the approximation uncorrelated vs. RPA or MFT vs. RHA one obtains substantially different results. The five to ten percent dispersion in $\mathcal{A}_{T}$ is larger than the accuracy needed to extract strange quark information.

For completeness we also show axial response functions and the $\mathcal{A}_{T^{\prime}}$ derived from them in Fig. 10. Since we have ignored strange-quark contributions, the axial response is purely isovector. Thus, (isoscalar) RPA correlations have no effect on the response and we only report uncorrelated results.

We see that the position and width of the quasielastic peak vary considerably reflecting the different effective masses of the various models. In addition, the finite nucleus calculations show their characteristic high-energy tails. Again, we observe differences of the order of 5 to 10 percent in the results.

Finally, in Figs. (11, 12, and 13) we simply plot all ten predictions for the total asymmetry $\mathcal{A}=\mathcal{A}_{L}+\mathcal{A}_{T}+\mathcal{A}_{T^{\prime}}$. These show the total dispersion in the different results. The experimental asymmetry is simply $a_{0} \tau$ times these curves. At $q=550 \mathrm{MeV} / \mathrm{c}$ and at an excitation energy of $\omega=150 \mathrm{MeV}$ the overall magnitude is $a_{0} \tau=-2.52 \times 10^{-5}$ (see also Table [V]).

\section{SUMMARY AND CONCLUSIONS}

In principle, a parity violation measurement in quasielastic electron scattering can provide information on strange quark contributions and radiative corrections to weak axial currents. This could greatly enhance the value of an elastic electron-proton experiment. However, in order for this to be useful one must understand nuclear structure corrections to the quasielastic measurement.

The quasielastic measurement is primarily sensitive to the ratio of isoscalar to isovector transverse responses $\mathcal{R}_{T}$. We have calculated these responses in a variety of relativistic mean field and RPA models for ${ }^{12} \mathrm{C}$ and nuclear matter at a momentum transfer of $q=550 \mathrm{MeV} / \mathrm{c}$. We find that $\mathcal{R}_{T}$ is sensitive to relativistic effects from a strong scalar field (small $M^{*}$ ) and from RPA correlations. Furthermore, full finite nucleus calculations predict large changes in $\mathcal{R}_{T}$ in the high energy tail of the response.

These nuclear structure effects could change the asymmetry $\mathcal{A}$ by as much as ten percent. This uncertainty is so large that a quasielastic measurement may not provide useful information on strange quark contributions or radiative corrections (unless these effects can be significantly constrained by future work). We note that a quasielastic measurement from deuterium rather than from a heavier nucleus should not be plagued by these uncertainties. We expect relativistic corrections to be much smaller for deuterium.

Alternatively, a quasielastic parity violating measurement could provide information on relativistic and nuclear structure effects on the isoscalar transverse response. This small response is interesting and not well determined by other data. This information might be obtained by comparing a deuterium measurement to that from a heavier nucleus such as ${ }^{12} \mathrm{C}$. 


\section{ACKNOWLEDGMENTS}

This research was supported by the Florida State University Supercomputer Computations Research Institute and U.S. Department of Energy contracts DE-FC05-85ER250000, DE-FG05-92ER40750, and DE-FG02-87ER40365.

\section{NUCLEON FORM FACTOR}

We adopt the form factor parameterization used in Ref. [5]. First the electromagnetic form factors are written in terms of a simple dipole,

$$
\begin{aligned}
G & =(1+4.97 \tau)^{-2}, \\
F_{1}^{(p)} & =\left[1+\tau\left(1+\lambda_{p}\right)\right] G /(1+\tau), \\
F_{2}^{(p)} & =\lambda_{p} G /(1+\tau), \\
F_{1}^{(n)} & =\tau \lambda_{n}(1-\eta) G /(1+\tau), \\
F_{2}^{(n)} & =\lambda_{n}(1+\tau \eta) G /(1+\tau) .
\end{aligned}
$$

Here the anomalous moments are,

$$
\lambda_{p}=1.793, \quad \lambda_{n}=-1.913
$$

and

$$
\eta=(1+5.6 \tau)^{-1}
$$

This parameterization is good for the neutron form factors provided $\tau \ll 1$. Finally, the isovector axial form factor is $\left(g_{a}=1.26\right)$

$$
G_{A}^{(3)}=\frac{g_{a}}{2}(1+3.53 \tau)^{-2} .
$$




\section{REFERENCES}

[1] M.J. Musolf and Barry R. Holstein, Phys. Lett. B242 (1990) 461.

[2] R.D. McKeown, Phys. Lett. B219 (1989) 140. Bates Experiment 89-06 (SAMPLE), R.D. McKeown and D.H. Beck spokesmen.

[3] L.A. Ahrens et al., Phys. Rev. D 35 (1987) 785.

[4] W. Heil et al., Nucl. Phys. B327 (1989) 1.

[5] T.W. Donnelly, M.J. Musolf, W.M. Alberico, M.B. Barbaro, A. De Pace and A. Molinari, Nucl. Phys. A541 (1992) 525.

[6] E. Hadjimichael, G.I. Poulis and T.W. Donnelly, MIT preprint 2041; submitted to Phys. Rev. C.

[7] N.K. Glendenning and S.A. Moszkowski, Phys. Rev. Lett. 67 (1991) 2414.

[8] Takumi Muto and Toshitaka Tatsumi, Phys. Lett. B283 (1992) 165.

[9] C.J. Horowitz, Phys. Rev. C in press.

[10] M.J. Musolf and T.W. Donnelly, MIT preprint 2041; submitted to Nucl. Phys. A.

[11] R. Rosenfelder, Ann. Phys. 128 (1980) 188.

[12] J.D. Walecka, Ann. Phys. (NY) 83 (1974) 491.

[13] B.D. Serot and J.D. Walecka, Advances in Nuclear Phys., Vol. 16, eds. J.W. Negele and E. Vogt (Plenum, NY, 1986).

[14] J.R. Shepard, E. Rost, C.-Y. Cheung and J.A. McNeil, Phys. Rev. C 37 (1988) 1130.

[15] K. Wehrberger and F. Beck, Phys. Rev. C 37 (1988) 1148.

[16] C.J. Horowitz and J. Piekarewicz, Phys. Rev. Lett. 62 (1989) 391; Nucl. Phys. A511 (1990) 461.

[17] J.R. Shepard, E. Rost and J.A. McNeil, Phys. Rev. C 40 (1989) 2320.

[18] C.J. Horowitz and B.D. Serot, Nucl. Phys A368 (1981) 503.

[19] C.J. Horowitz and B.D. Serot, Phys. Lett. B140 (1984) 181.

[20] J.A. McNeil, R.D. Amado, C.J. Horowitz, M. Oka, J.R. Shepard and D.A. Sparrow, Phys. Rev. C 34 (1986) 746.

[21] R.J. Furnstahl and B.D. Serot, Nucl. Phys. A468 (1987) 539.

[22] C.J. Horowitz, Phys. Lett. B208 (1988) 8.

[23] H. Kurasawa and T. Suzuki, Nucl. Phys. A490 (1988) 571.

[24] Z.E. Meziani et al., Phys. Rev. Lett 52 (1984) 2130. 


\section{FIGURES}

FIG. 1. Transverse response as a function of energy loss for ${ }^{12} \mathrm{C}$ at $q=550 \mathrm{MeV}$. The solid (dot-dashed) line gives the uncorrelated (RPA) Fermi gas result. The dashed (dotted) line gives the uncorrelated (RPA) result using an effective mass of $M^{*}=0.68$. All calculations ignore vacuum polarization and used a Fermi momentum of $k_{F}=225 \mathrm{MeV}$. Experimental data is from Ref. [24].

FIG. 2. Same as Fig. 1 but for the isoscalar contribution to the transverse response.

FIG. 3. Same as Fig. 目 but for: (a) the transverse isoscalar to isovector ratio and (b) the transverse contribution to the parity-violating asymmetry at $\theta=150^{\circ}$.

FIG. 4. Transverse response as a function of energy loss for ${ }^{12} \mathrm{C}$ at $q=550 \mathrm{MeV}$. The solid (dot-dashed) line gives the uncorrelated (RPA) finite-nucleus result. The dashed (dotted) line gives the uncorrelated (RPA) result using an effective mass of $M^{*}=0.68$. All calculations ignore vacuum polarization and (the nuclear matter ones) used a Fermi momentum of $k_{F}=225 \mathrm{MeV}$. Experimental data is from Ref. [24].

FIG. 5. Same as Fig. 团 but for the isoscalar contribution to the transverse response.

FIG. 6. Same as Fig. 1 but for: (a) the transverse isoscalar to isovector ratio and (b) the transverse contribution to the parity-violating asymmetry at $\theta=150^{\circ}$.

FIG. 7. Transverse response as a function of energy loss for ${ }^{12} \mathrm{C}$ at $q=550 \mathrm{MeV}$. The solid (dot-dashed) line gives the uncorrelated (RPA) finite-nucleus result. The dashed (dotted) line gives the uncorrelated (RPA) result using an effective mass of $M^{*}=0.80$. All calculations include vacuum polarization and (the nuclear matter ones) used a Fermi momentum of $k_{F}=225 \mathrm{MeV}$. Experimental data is from Ref. [24].

FIG. 8. Same as Fig. 7 but for the isoscalar contribution to the transverse response.

FIG. 9. Same as Fig. 7 but for: (a) the transverse isoscalar to isovector ratio and (b) the transverse contribution to the parity-violating asymmetry at $\theta=150^{\circ}$.

FIG. 10. Axial isovector response (a), and its contribution to the parity-violating asymmetry at $\theta=150^{\circ}$ (b), as a function of energy loss for ${ }^{12} \mathrm{C}$ at $q=550 \mathrm{MeV}$. The solid (dot-dashed) line gives the MFT (RHA) finite-nucleus result. The dashed (dotted) line gives the MFT (RHA) nuclear matter result. Also shown are free Fermi gas results. Nuclear matter calculations used a Fermi momentum of $k_{F}=225 \mathrm{MeV}$. 
FIG. 11. Parity-violating asymmetry as a function of energy loss for ${ }^{12} \mathrm{C}$ at $\theta=150^{\circ}$ and $q=550 \mathrm{MeV}$. The solid (dot-dashed) line gives the uncorrelated (RPA) Fermi gas result. The dashed (dotted) line gives the uncorrelated (RPA) result using an effective mass of $M^{*}=0.68$. All calculations ignore vacuum polarization and used a Fermi momentum of $k_{F}=225 \mathrm{MeV}$.

FIG. 12. Parity-violating asymmetry as a function of energy loss for ${ }^{12} \mathrm{C}$ at $\theta=150^{\circ}$ and $q=550 \mathrm{MeV}$. The solid (dot-dashed) line gives the uncorrelated (RPA) finite-nucleus result. The dashed (dotted) line gives the uncorrelated (RPA) result using an effective mass of $M^{*}=0.68$. All calculations ignore vacuum polarization and (the nuclear matter ones) used a Fermi momentum of $k_{F}=225 \mathrm{MeV}$.

FIG. 13. Parity-violating asymmetry as a function of energy loss for ${ }^{12} \mathrm{C}$ at $\theta=150^{\circ}$ and $q=550 \mathrm{MeV}$. The solid (dot-dashed) line gives the uncorrelated (RPA) finite-nucleus result. The dashed (dotted) line gives the uncorrelated (RPA) result using an effective mass of $M^{*}=0.80$. All calculations include vacuum polarization and (the nuclear matter ones) used a Fermi momentum of $k_{F}=225 \mathrm{MeV}$. 


\section{TABLES}

TABLE I. Electro-weak charges for electrons and $u, d$, and, $s$ quarks. Numerical values were evaluated using $\sin ^{2} \theta_{W} \simeq 0.227$.

\begin{tabular}{cccc}
\hline \hline Fermion & $e(E M)$ & $g_{V}($ Weak Vector $)$ & $g_{A}($ Weak Axial $)$ \\
\hline$e$ & -1 & $-1+4 \sin ^{2} \theta_{W} \simeq-0.091$ & +1 \\
$u$ & $+2 / 3$ & $+1-\frac{8}{3} \sin ^{2} \theta_{W} \simeq+0.394$ & -1 \\
$d$ & $-1 / 3$ & $-1+\frac{4}{3} \sin ^{2} \theta_{W} \simeq-0.697$ & +1 \\
$s$ & $-1 / 3$ & $-1+\frac{4}{3} \sin ^{2} \theta_{W} \simeq-0.697$ & +1 \\
\hline \hline
\end{tabular}

TABLE II. Isoscalar, isovector, and strange (or singlet) weak-vector and weak-axial couplings. Numerical values were evaluated using $\sin ^{2} \theta_{W} \simeq 0.227$.

\begin{tabular}{ccc}
\hline \hline Coupling & (Weak Vector) & (Weak Axial) \\
\hline$\sqrt{3} \xi^{(0)}$ & $-4 \sin ^{2} \theta_{W} \simeq-0.909$ & 0 \\
$\xi^{(1)}$ & $2-4 \sin ^{2} \theta_{W} \simeq+1.091$ & -2 \\
$\xi^{(s)}$ & -1 & +1 \\
\hline \hline
\end{tabular}

TABLE III. Coupling constants, meson masses, and effective nucleon mass in a mean-field approximation to the Walecka model. The effective nucleon mass was calculated using a Fermi momentum of $k_{\mathrm{F}}=225 \mathrm{MeV}$.

\begin{tabular}{lccccc}
\hline \hline Model & $g_{\mathrm{s}}^{2}$ & $g_{\mathrm{v}}^{2}$ & $m_{\mathrm{s}}(\mathrm{MeV})$ & $m_{\mathrm{v}}(\mathrm{MeV})$ & $M^{*} / M$ \\
\hline MFT & 109.626 & 190.431 & 520 & 783 & 0.68 \\
RHA & 54.289 & 102.770 & 458 & 783 & 0.80 \\
\hline \hline
\end{tabular}

TABLE IV. Ratio of isoscalar to isovector transverse responses and parity-violating asymmetries for ${ }^{12} \mathrm{C}$ using various nuclear-structure models at $q=550 \mathrm{MeV}, \omega=150 \mathrm{MeV}$, and $\theta=150^{\circ}$. The nuclear matter calculations used a Fermi momentum of $k_{\mathrm{F}}=225 \mathrm{MeV}$. Within each model, the first (second) row of numbers give the uncorrelated (RPA) results.

\begin{tabular}{lcccc}
\hline \hline Model & $\mathcal{R}_{T}$ & $\mathcal{A}_{T} / a_{0} \tau$ & $\mathcal{A}_{T^{\prime}} / a_{0} \tau$ & $\mathcal{A} / a_{0} \tau$ \\
\hline Free Fermi Gas & 0.044 & 0.973 & 0.198 & 1.168 \\
& 0.030 & 1.000 & 0.201 & 1.201 \\
\hline MFT $\left[M^{*}=0.68\right]$ & 0.083 & 0.915 & 0.180 & 1.090 \\
& 0.071 & 0.937 & 0.182 & 1.117 \\
\hline MFT [Fin. Nuc.] & 0.072 & 0.928 & 0.184 & 1.107 \\
& 0.068 & 0.937 & 0.185 & 1.119 \\
\hline RHA[ $\left.M^{*}=0.80\right]$ & 0.064 & 0.943 & 0.188 & 1.126 \\
& 0.039 & 0.994 & 0.194 & 1.191 \\
\hline RHA [Fin. Nuc.] & 0.054 & 0.957 & 0.192 & 1.144 \\
& 0.051 & 0.969 & 0.194 & 1.164 \\
\hline \hline
\end{tabular}

